Herz 2013 · 38:695-695

DOI 10.1007/s00059-013-3973-1

Online publiziert: 27. September 2013

(c) Urban \& Vogel 2013

R. Zahn

Abt. Kardiologie, Herzzentrum Ludwigshafen , Ludwigshafen

\title{
Karotisinterventionen: Pro und Kontra
}

gestellt. In den Artikeln von Storck und Schofer/Bijuklic wird dann auf die technische Durchführung der CEA und des CAS eingegangen, die jeweils ausführlich dargestellt werden. Der Beitrag von HeinRothweiler und Mudra gibt einen Überblick über die verschiedenen Leitlinien zur Therapie der Karotisstenosen und versucht, die Unterschiede in der Bewertung des CAS zu erklären. Staubach und $\mathrm{Mu}$ dra zeigen in ihrem Artikel, dass wie für alle komplexeren Interventionen wie etwa auch für die perkutane Koronarintervention (PCI; $[7,8]$ ) auch für das CAS ein Zusammenhang zwischen der Erfahrung des Untersuchers und den Ergebnissen besteht. Im Beitrag von Werner wird gezeigt, dass auch im Zeitalter von gesetzlich vorgeschriebener Qualitätssicherung noch Bedarf für Register besteht und diese doch deutlich über die Möglichkeiten der reinen Qualitätssicherung hinausgehen. Zusammenfassend kann festgehalten werden, dass das CAS eine gleichwertige Alternative zur CEA bei symptomatischen und asymptomatischen Stenosen der A. carotis darstellt. Neben einer interdisziplinären Indikationsstellung, am besten in einem vaskulären Arbeitskreis mit Neurologen, Interventionalisten und Chirurgen, sollten auch lokale Gegebenheiten wie Erfahrung der Untersucher und Ergebnisse der Interventionen berücksichtigt werden.

\section{RalfZahn}

\section{Korrespondenzadresse}

Prof. Dr. R. Zahn

Abt. Kardiologie,

Herzzentrum Ludwigshafen

Bremserstraße 79, 67063 Ludwigshafen

erzahn@aol.com

\section{Einhaltung ethischer Richtlinien}

Interessenkonflikt. R. Zahn gibt an, dass kein Interessenkonflikt besteht.

Dieser Beitrag beinhaltet keine Studien an Menschen oder Tieren.

\section{Literatur}

1. Norris JW, Zhu CZ, Bornstein NM et al (1991) Vascular risks of asymptomatic carotid stenosis. Stroke 22:1485-1490

2. North American Symptomatic Carotid Endarterectomy Trial Collaborators (1991) Beneficial effect of carotid endarterectomy in symptomatic patients with high-grade carotid stenosis. N Engl J Med 325:445-453

3. European Carotid Surgery Trialists'Collaborative Group (1998) Randomised trial of endarterectomy for recently symptomatic carotid stenosis: final results of the MRC European Carotid Surgery Trial (ECST). Lancet 351:1379-1387

4. Halliday A, Mansfield A, Marro J et al (2004) Prevention of disabling and fatal strokes by successful carotid endarterectomy in patients without recent neurological symptoms: randomised controlled trial. Lancet 363:1491-1502

5. Mathias K (1987) Catheter treatment of arterial occlusive disease of supraaortic vessels. Radiologe 27:547-554

6. Mathias K, Gospos C, Thron A et al (1980) Percutaneous transluminal treatment of supraaortic artery obstruction. Ann Radiol (Paris) 23:281-282

7. Zahn R, Gottwik M, Hochadel M et al (2008) Volume-outcome relation for contemporary percutaneous coronary interventions ( $\mathrm{PCl}$ ) in daily clinical practice: is it limited to high-risk patients? Results from the Registry of Percutaneous Coronary Interventions of the Arbeitsgemeinschaft Leitende Kardiologische Krankenhausärzte (ALKK). Heart 94:329-335

8. Harold JG, Bass TA, Bashore TM et al (2013) ACCF/ AHA/SCAI 2013 Update of the Clinical Competence Statement on Coronary Artery Interventional Procedures: A Report of the American College of Cardiology Foundation/American Heart Association/American College of Physicians Task Force on Clinical Competence and Training (Writing Committee to Revise the 2007 Clinical Competence Statement on Cardiac Interventional Procedures). Circulation 128:436-472 orikationsstellung und zum Ablauf von Karotisinterventionen besprochen. Außerdem wird die neurologische Sicht dar- 